

\title{
Editorial
}

\section{Building paramedics, maintaining skilled practitioners}

\author{
Jean-Paul Veronese BT(Emergency Medical Care), MSc(Emergency Medicine), is a Lecturer in Paramedicine ${ }^{1}$
}

\author{
Affiliation: \\ ${ }^{1}$ School of Medicine, Griffith University, Queensland
}

Building and maintaining skilled paramedics is a key component within the health system. Most paramedics never forget their first day as a new graduate or newly qualified paramedic, often with mixed feelings about being 'unleashed on the world'. Many new paramedics will be given the keys to a freshly stocked ambulance and be expected to attend to a vast array of calls ranging from minor to challenging. My own experience was not different to most others, with the first day consisting of two paediatric drowning resuscitations an hour apart followed by a motor vehicle accident entrapment and a fall from height with spinal injury. For the most part, these calls went surprisingly well. However, for those parts that didn't go as well or could have been better, the clinician is often left with thoughts of 'what if' and 'if only I had...'. Herein lies the importance of quality education followed by an appropriate work environment that fosters good practice.

Medical education is typically delivered across health sciences faculties within higher education institutions. Departments that have traditionally fallen under the banner of health science education include medicine and allied health professions such as nursing and physiotherapy, with paramedicine one of the latest disciplines to join the professions. Despite paramedicine being relatively new by comparison - only having been around for several decades - as a profession it has grown rapidly. However, despite its rapid growth there remain some areas that reflect this infancy. One area is the variation in practice and educational standards, both locally and internationally.

With this in mind, building clinicians to work in these various environments remains a challenge. Medical education has recognised for years that several factors influence the successful performance of any competency. These include the learner's knowledge of what is required, the component skills required to accomplish this, the correct attitude that displays a confidence and willingness to do what is required, and maintaining a professional environment that supports appropriate behaviour. These core components have been summarised into a taxonomy of learning domains that is still widely recognised today. These components include the cognitive, affective and psychomotor domains.

The cognitive domain involves adult knowledge acquisition and development of intellectual skills. Bloom's taxonomy outlines how learners ascend through the levels of intellectual skill, from simple recall of information through to evaluating and creating new information (1). Similar models include Bigg's SOLO taxonomy, which describes levels of increasing complexity in a learner's understanding of subjects (2). These and other models are used as means of learning, teaching and assessment of the cognitive domain. Teaching strategies are generally comprised of a multi-modal approach, which has been outlined in a previous editorial within this journal (3). While these have proven to be effective models to work with, the execution becomes more of a fine juggling act: balancing the right amount of information needed by the learners, while not overloading them.

The psychomotor domain involves adult skill acquisition. Simply put, this is comprised of physical movement, coordination and use of the motor-skill areas. Several models are widely used throughout medical education the including Simpson (4), Harrow (5) and Dreyfus (6) models of skill acquisition. Traditionally, medical education relied on the Halstedian mantra 'see one, do one, teach one', but this has lost favour due to concerns with patient safety (7). Simulation-based training has been offered as a replacement for practising on patients and has widely been accepted as the new method of teaching skills (8). Paramedicine learners progress from performing simple tasks such as oropharyngeal airway insertion in the form of an isolated clinical skill, through to more complex simulation-based scenarios involving critically ill and injured patients in the simulated setting. Learners are expected to achieve competency level within these models, and only progress to proficiency and mastery level after several years of practice as qualified clinicians. Assessing competency can also prove to be a challenging process in education, as assessment rubrics traditionally possess outcomes that may or may not always be reflective of the learner's competency level. Traditional rubrics also generally possess very stringent quantitative means of assessment. Assessment rubrics that possess both aspects of qualitative and quantitative measures should be considered to gauge an overall perspective of skill competency, as well as possessing characteristics of reliability and validity. 
Even with skill acquisition being more reliant on simulation-based teaching, a key component to completing the learner's competency is performing skills on patients under direct supervision. A learner who has practised their skills sufficiently and is competent in the simulated setting should be well prepared to perform learned skills on patients under direct supervision. This is a key transition point for the learner. Learners must also achieve a set number of skills to safely practise autonomously after they have graduated and qualified. Several challenges exist with attaining these numbers, including low frequency of opportunities or cases to perform these skills, or supervisors who are unwilling to allow learners to 'practise' on their licence for fear of reprisal.

Once competency is achieved, skill decay - or 'de-skilling' - can occur over time if the skill is not performed frequently. This occurs faster among novice clinicians than it does among the more experienced. With the varying nature of calls attended by paramedics, the opportunity to stagger the level of skill decay through practise becomes a predicament not only for the paramedic, but the patients they attend. Systems can ensure patient and practitioner safety by implementing robust continuous quality improvement systems, which have been shown to improve outcomes (9-11). Simulation-based interventions to avoid skill decay could be a solution to this, and remains an active area of research. Ultimately, the journey from paramedic learner to graduate should be one of constant learning, with robust systems in place to support this process. These systems are vital in ensuring the health and safety of both the patient and the paramedic. The old adage 'rescuer-safety first' should always remain our priority.

Jean-Paul Veronese

Griffith University, Queensland

\section{References}

1. Bloom Engelhardt MD, Furst EJ, Hill WH, Krathwol DRBS. A taxonomy of educational objectives. Handbook 1, the cognitive domain. New York: David McKay Co; 1956.

2. Biggs J, Collis KF. Evaluating the quality of learning: the SOLO taxonomy. A Handbook for Teaching and Learning in Higher Education: Enhancing Academic Practice 1982, p. 13.

3. Smith $\mathrm{G}$. The challenges of paramedic education in the new millennium: Chasing the evolution of paramedic practice. Australasian Journal of Paramedicine 2017;14(4).

4. Simpson EJ. The classification of educational objectives in the psychomotor domain. Education 1972;3:43-56. Available at: http://eric.ed.gov/ERICWebPortal/recordDetail?accno=ED010368

5. Harrow A. A taxonomy of the psychomotor domain: a guide for developing behavior. New York. 1972, p. 36-76. Available at: https://www.researchgate.net/publication/31840399_A_Taxonomy_of_the_Psychomotor_Domain_A_Guide_for_Developing_ Behavioral_Objectives_AJ_Harrow

6. Dreyfus SE. The five-stage model of adult skill acquisition. Bull Sci Technol Soc 2004;24:177-81.

7. Ziv A, Root Wolpe P, Small SD, Glick S. Simulation-based medical education: an ethical imperative. Acad Med 2003;78:783-8.

8. Lenchus JD. End of the "see one, do one, teach one" era: the next generation of invasive bedside procedural instruction. J Am Osteopath Assoc 2010;110:340-6.

9. Nestler DM, Noheria A, Haro LH, et al. Sustaining improvement in door-to-balloon time over 4 years: the Mayo clinic STelevation myocardial infarction protocol. Circ Cardiovasc Qual Outcomes 2009;2:508-13. Available at: www.ncbi.nlm.nih.gov/ pubmed/20031884

10. Santana MJ, Stelfox HT. Quality indicators used by trauma centers for performance measurement. J Trauma Acute Care Surg 2012;72:1298-302; Discussion 12303. Available at: www.ncbi.nlm.nih.gov/pubmed/22673258

11. Jollis JG, Granger CB, Henry TD, et al. Systems of care for ST-segment-elevation myocardial infarction: a report From the American Heart Association's Mission: Lifeline. Circ Cardiovasc Qual Outcomes 2012;5:423-8. Available at: www.ncbi.nlm.nih. gov/pubmed/22619274 